\title{
LEUKOCYTOSIS FOLLOWING THE INTRAMUSCULAR INJECTION OF LIVER EXTRACT
}

\author{
By JOHN H. POWERS AND WILLIAM P. MURPHY \\ WITH THE ASSISTANCE OF KATHARINE HUMPHREYS \\ (From the Department of Surgery of the Mary Imogene Bassett Hospital, \\ Cooperstown, New York)
}

(Received for publication April 19, 1933)

The observations submitted in this paper record the effect of the intramuscular injection of liver extract on the total number of white cells and polymorphonuclear neutrophiles in the peripheral circulating blood of normal individuals.

The stimulating effect of the oral ingestion of whole liver on the formed elements of the blood other than the red cells and the reticulocytes was first recognized by Murphy, Monroe and Fitz (1). These observations were substantiated by Minot and Murphy (2) in a large group of patients with pernicious anemia. In a more recent paper on the parenteral use of liver extract in pernicious anemia Murphy (3) recorded "a prompt and marked increase" in the number of polymorphonuclear neutrophiles within a few hours after the intramuscular injection of an extract prepared by Lederle Laboratories, Inc.

The experimental work herein reported was undertaken to determine the effect of injections of liver extract on the leukocytic count of normal subjects. Should it prove possible to stimulate a leukocytosis by this procedure in normal individuals, it was hoped that the observation might be utilized clinically in the treatment of patients with chronic sepsis and a persistently low level of leukocytes.

\section{METHODS OF STUDY}

The subjects were members of the visiting, house, and laboratory staffs or convalescent surgical patients on the wards of the Mary Imogene Bassett Hospital. The age of the individuals varied from 6 years to 72 years.

Blood for each count was taken from the finger into a standardized pipette, diluted, shaken for 3 minutes and counted immediately in a standardized Levy counting chamber. (Both pipettes and counting chambers were certified by the U. S. Bureau of Standards.) Smears were made from 1 drop of blood spread in a thin film on a glass slide and stained with Wright's stain. Differential counts were taken from an average of 200 cells.

A series of observations covering 3 consecutive days was made on 
each of 22 subjects. The first day of the experiment was used as a control period in order to obtain the normal daily range of the leukocytic level. The individuals were divided into 2 groups, to the first of which liver extract was given at 9 o'clock in the forenoon and to the second of which the drug was administered at 5 o'clock in the morning.

In the first group, blood for counts was taken at 9 and 11 o'clock in the forenoon, 2 and 4 in the afternoon. At 8 o'clock on the morning of the second day, an additional control count was taken. At 9 o'clock, 3 cc. of liver extract ${ }^{1}$ were given intramuscularly in the gluteal region. Complete determinations were then made hourly until 7 o'clock in the evening. On the third day counts were taken at 9 and 11 in the forenoon, 2 and 4 in the afternoon. Thus it was possible not only to obtain a curve of the leukocytic level at hourly intervals for 10 hours after the intramuscular injection of liver extract, but also to have for comparison with this curve observations made at definite intervals throughout the day preceding and the day following the experiment.

In the second group, specimens of blood for control counts during the first day were taken at 6 and 9 o'clock in the morning, 12 noon, and 3 in the afternoon. An additional control count was made at 5 o'clock on the morning of the second day and 3 cc. of liver extract were given intramuscularly immediately thereafter. Complete determinations were then made every hour until 5 o'clock in the afternoon. In this group, only one control count was made during the third day, at 9 o'clock in the morning.

\section{RESULTS}

Observations were made on 22 individuals. There were 17 in the first group, to whom liver extract was given at 9 o'clock in the forenoon, and 5 in the second group who received the same amount of extract 4 hours earlier, at 5 o'clock in the morning. For the purpose of analysis and comparison, the first group has been further divided into 2 subgroups, ambulatory and recumbent.

In the ambulatory group are included 7 individuals, all normal, who went about their daily duties in the hospital during the course of the experiment. The results have been averaged and are presented graphically in Figure 1. The greatest individual increase in the total number of white cells in this group amounted to 185 per cent and the smallest was 23 per cent above the average of the 4 control counts made on each of these 2 subjects the previous day. The highest average increase in all 7 cases was 94.1 per cent. The peak of the rise occurred 7 hours after the injection of liver extract and was partially maintained for several hours longer. This increase in the total number of white cells in the peripheral blood was due to both an actual and a relative increase in the total number of polymorpho-

1 This extract was prepared by the Lederle Laboratories, Inc. and furnished through the kindness of Dr. Guy W. Clark. 
nuclear neutrophiles. The curve of the neutrophiles follows closely and stamps its own characteristics upon that of the total white cells (Figure 1). The percentage of neutrophilic leukocytes rose from an average of 62 on the first day of the experiment to a peak of 77 five hours after the administration of liver extract.

The recumbent group includes 9 patients convalescing from fractures

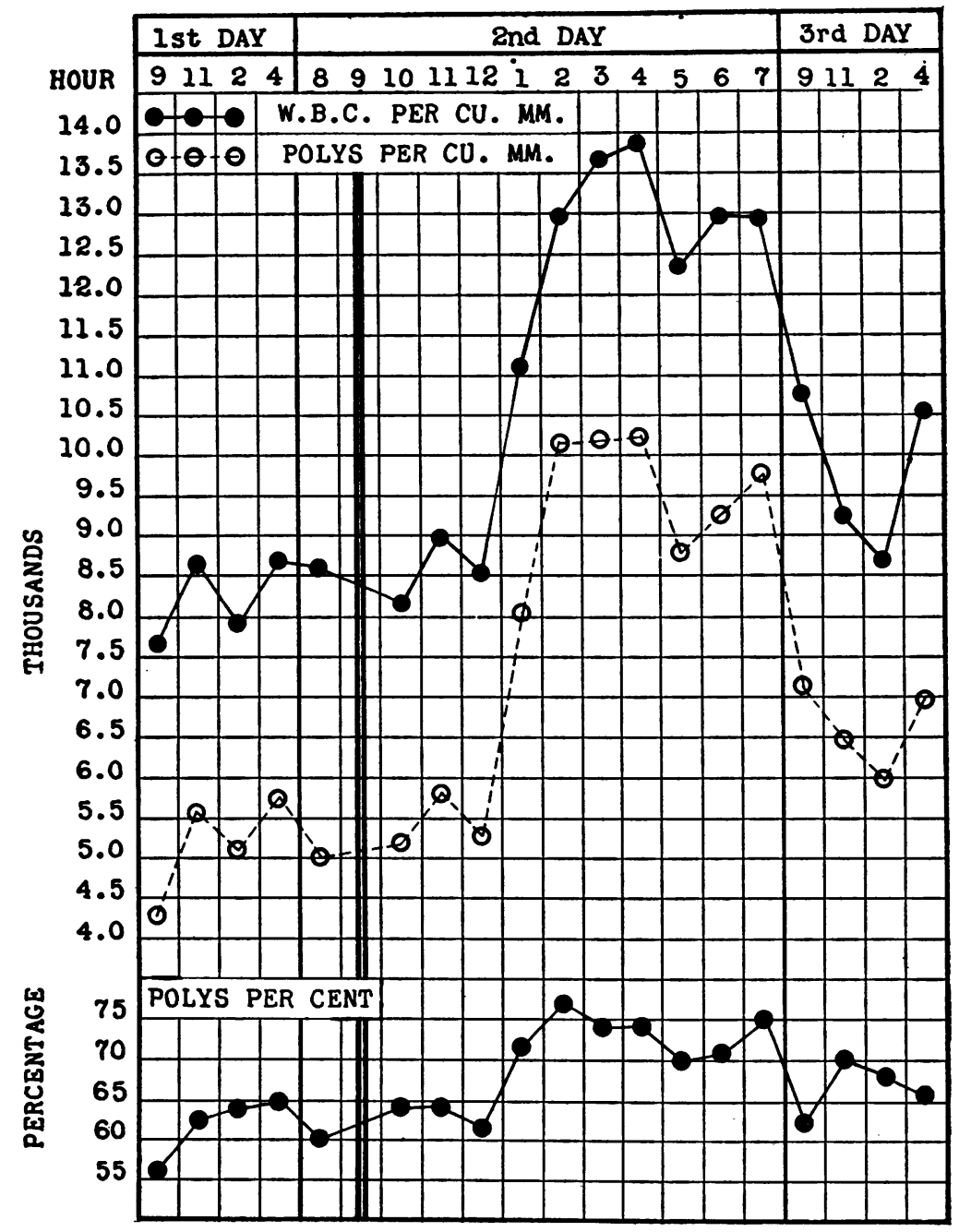

Fig. 1. A Composite Graph of the Total White Cells and the Total Polymorphonuclear Neutrophiles per Cubic Millimeter and the Percentage of Polymorphonuclear Neutrophiles of Seven Ambulatory, Normal Subjects.

The single vertical lines separate the chart into days; the double vertical line represents the hour at which $3 \mathrm{cc}$. of liver extract were given intramuscularly. 
or minor surgical operations. All were confined to bed during the progress of the experiment. These results have been averaged and are presented as a composite graph in Figure 2. The greatest average increase in total white cells following the administration of liver extract was 72.0 per cent higher than the average of all control counts. The highest individual

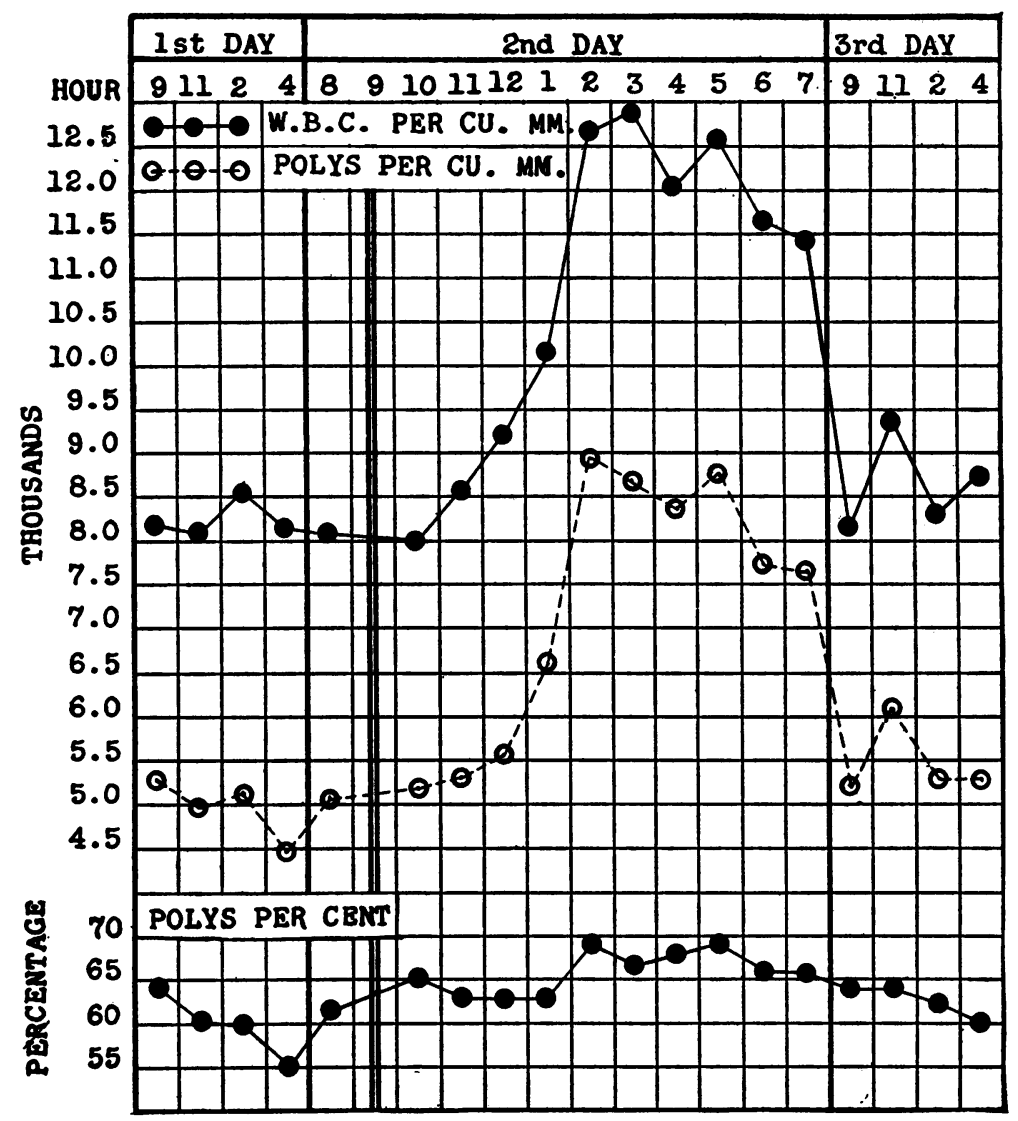

Fig. 2. A Composite Graph of the Total White Cells and the Total Polymorphon Uclear Neutrophiles per Cubic Millimeter and the Percentage of Polymorphonuclear Neutrophiles of Nine Recumbent Convalescent, Surgical Patients with No Infectious Process.

The single vertical lines separate the chart into days; the double vertical line represents the hour at which 3 cc. of liver extract were given intramuscularly.

increase was 101 per cent and the lowest was 20 per cent greater than the average counts of these 2 patients made the previous day. Again, the increase in total white cells was due both to greater actual numbers and to higher percentage of polymorphonuclear neutrophiles. The curve of the latter is almost a replica of that of the former (Figure 2). 
One house officer in this group who volunteered as a subject for this experimental work came down with influenza during the first day of observation and the results are presented separately in Figure 3 . The development of leukopenia during the control period was interesting. The subsequent response of total white cells, total number and percentage of polymorphonuclear neutrophiles after the intramuscular injection of liver extract was comparable to that obtained in normal subjects.

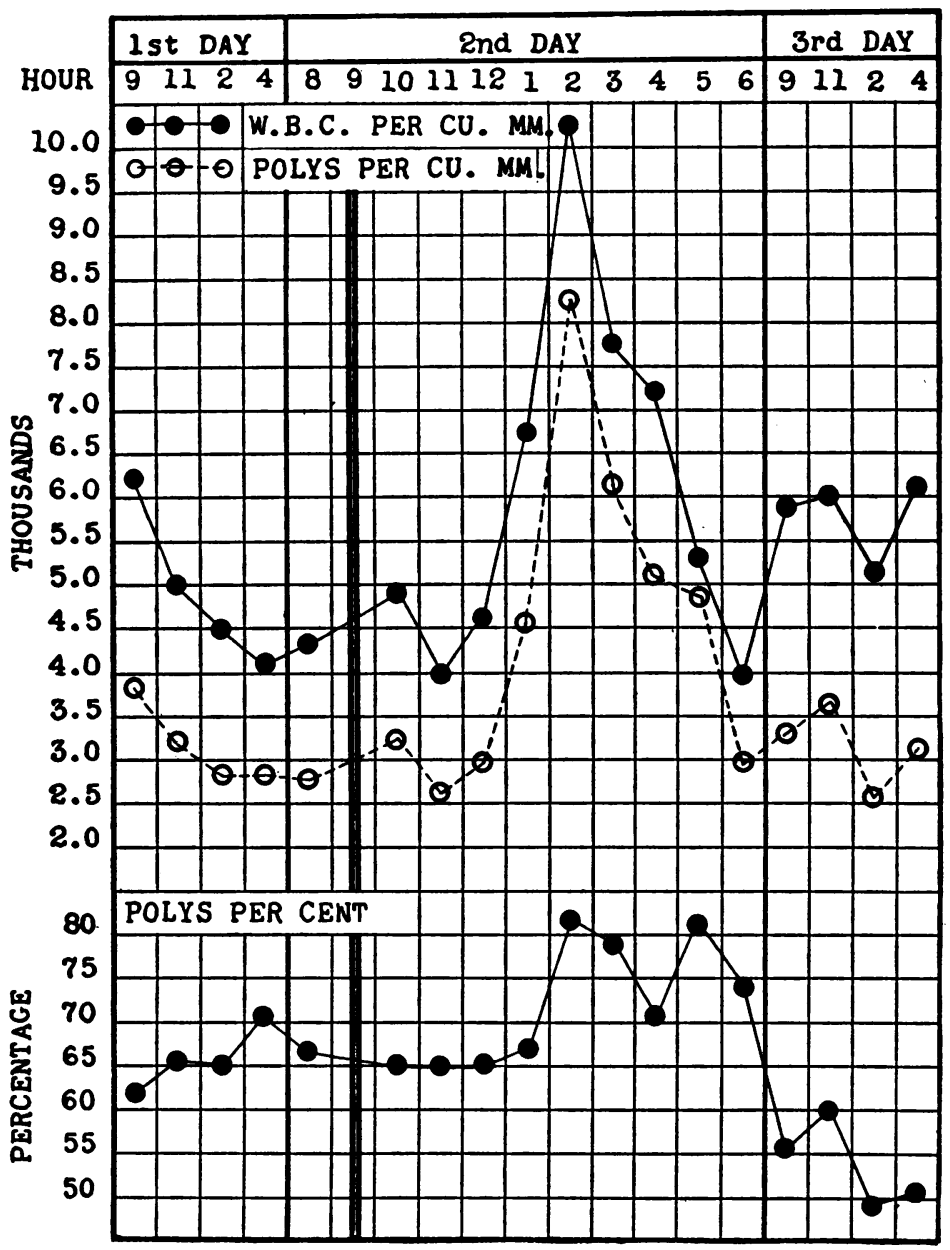

Fig. 3. A Graph of the Total White Cells and the Total PolyMorphonuclear Neutrophiles Per Cubic Millimeter and the Percentage of Polymorphonuclear Neutrophiles of a House Officer Who Came Down with Influenza on the First Day of the Experiment.

The onset of leukopenia is apparent in the four observations recorded on this day. The response following the intramuscular administration of liver extract is comparable to that presented in Figures 1 and 2 for normal subjects. 
The second major group includes 5 subjects to whom liver extract was given at 5 o'clock in the morning. Two of these individuals were ambulatory house officers and 3 were recumbent patients who presented no demonstrable evidence of infection. The response in all the members of

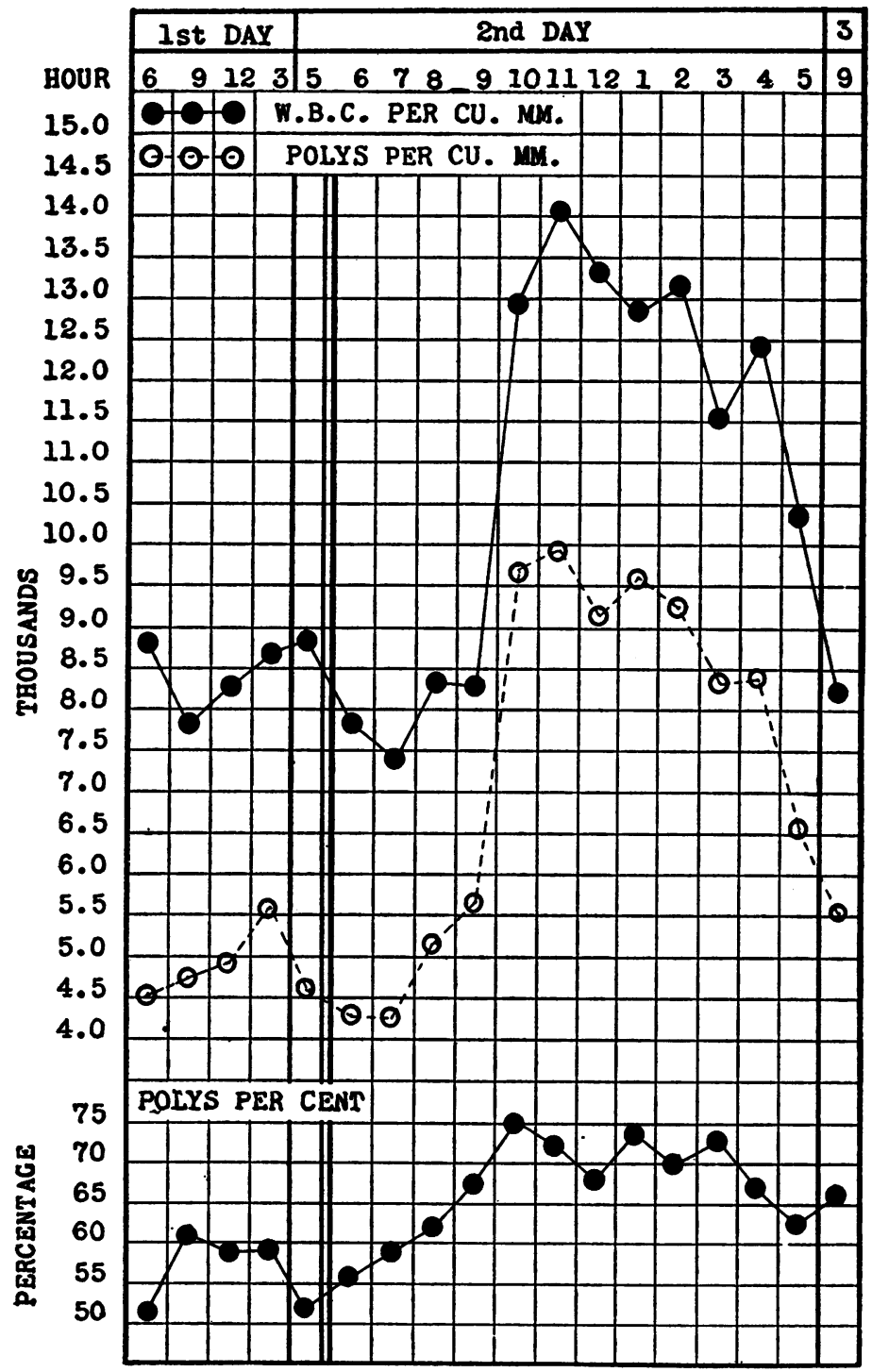

Fig. 4. A Composite Graph of the Total White Cells, the Total Number and Percentage of Polymorphonuclear Neutrophiles of Five Ambulatory and Recumbent Subjects to Whom Intramuscular Liver Extract Was Given at Five O’Clock in the Morning.

The maximal response occurred 6 hours later and is similar to that which followed injection of the extract at nine o'clock (compare Figures 1 and 2). 
this group was similar to that recorded for the individuals embraced in both the sub-divisions of the first group. The greatest average increase in total white cells was 71 per cent higher than the average of all control counts made during the previous day. The maximal and minimal individual responses were 85 per cent and 60 per cent respectively. The percentage of polymorphonuclear neutrophiles rose from an average of 57 to a peak of 75 . A composite graph of the results is presented in Figure 4.

\section{DISCUSSION}

These observations indicate an increase in the total number of white cells and the total number and percentage of polymorphonuclear neutrophiles in the peripheral circulating blood of 21 ambulatory or recumbent normal subjects and 1 patient with influenza following the intramuscular injection of $3 \mathrm{cc}$. of liver extract. The response begins in 2 hours and reaches a peak in 6 or 7 hours after the injection.

Numerous investigators have established the fact that the normal leukocyte count in health may be an extremely variable figure. In a series of observations made every 15 minutes on subjects both at rest and ambulatory, with and without food, Sabin et al. (4) demonstrated oscillations which in some cases seemed to pursue a certain hourly rhythm with an upward trend of the peaks in the late afternoon and a normal variation as high as $1: 2$. Shaw (5) has demonstrated "diurnal tides" in the normal ebb and flow of the leukocytes which reach their peak about 5 o'clock in the afternoon and 5 o'clock in the morning with low points between 10 and 12 noon and 9 and 11 in the evening. He believes that the protagonists of the theory of "digestion leukocytosis" have in reality been describing, as a physiological response to food, the normal afternoon flow of the leukocytic tide. He also offers experimental observations to support the belief that the "pursuit of an ordinary day's activity with its concomitant physical variations produces no gross departure from the leukocyte curve of rest and starvation."

Other investigators have demonstrated changes in the total number of white blood cells after the injection of drugs and foreign substances, the ingestion of food, stimulation of nerves, changes in temperature and blood pressure, exercise, and even alterations in posture. None of the subjects included in the experimental work herein reported received any stimulating drugs other than liver extract during the period of observation. The amount of foreign protein in the extract, if present at all, is infinitesimal. ${ }^{2}$ All took their usual meals at the customary hours. The members of the ambulatory group were subjected to the changes in environmental tempera-

2 In a personal communication to the authors Dr. Clark of the Lederle Laboratories, Inc., states "our commercial liver extract is in all respects a proteinfree preparation." 
ture and muscular activity which occur in the normal routine of a day on the wards and in the laboratories of a hospital. The recumbent patients remained at rest in bed, exposed only to the slight hourly fluctuations in the temperature of the ward.

All the details of the external environment were essentially comparable for the subjects of each group during the 3 days of the experiment. The number of total white cells and polymorphonuclear neutrophiles remained fairly constant at representative hours during the first and third days, which were utilized as controls. Hence it seems reasonable to assume that the leukocytosis and both the actual and relative increase in the number of neutrophiles, which followed the intramuscular injection of liver extract on the second day, may be attributed to the effect of this substance.

Further support of this contention is offered by the results obtained in the second group of subjects and presented graphically in Figure 4. When liver extract was injected at 5 o'clock in the morning the maximal leukocytic response occurred at 11 o'clock, the approximate hour at which, according to Shaw, the normal diurnal leukocytic tide is at its lowest ebb. Hence, it cannot be argued that the maximal responses of the first group, which occurred at 3 and 4 o'clock in the afternoon, were due to the normal physiological flood of this same diurnal tide.

\section{SUM MARY}

- Determinations of the total white cells and the total number and percentage of polymorphonuclear neutrophiles in the peripheral circulating blood of 21 normal subjects before and after the intramuscular injection of liver extract have been presented in graphic form. The individuals were divided into 2 groups, those to whom the extract was given at 9 o'clock in the morning, and those who received the drug at 5 o'clock. The first group was further divided in 2 sub-groups, ambulatory and recumbent.

The average maximal increase in the total number of white cells of the ambulatory group was 94.1 per cent higher than the average of all control counts and occurred 7 hours after the extract was administered. The greatest individual response in this group was 185 per cent and the lowest was 23 per cent above the average of the 4 control counts made on each of these 2 subjects the previous day.

The average maximal increase in total white cells of the patients of the recumbent group was 72 per cent above the normal level and occurred 6 hours after the injection of liver extract. The highest and lowest individual responses in the members of this group were 101 per cent and 20 per cent above the normal averages of these two subjects.

One patient with influenza, accompanied by leukopenia on the first day or the experiment, showed a well marked leukocytosis following the injection of liver extract.

A similar increase in the total number of white cells and the total num- 
ber and percentage of polymorphonuclear neutrophiles was obtained in the second group of normal subjects who received the drug 4 hours earlier than those of the first group.

The leukocytosis in every instance was due to an increase in the polymorphonuclear neutrophiles.

\section{BIBLIOGRAPHY}

1. Murphy, W. P., Monroe, R. T., and Fitz, R., J. A. M. A., 1927, 1xxxviii, 1211. Changes in Composition of Blood in Pernicious Anemia.

2. Minot, G. R., and Murphy, W. P., J. A. M. A., 1927, lxxxix, 759. A Diet Rich in Liver in the Treatment of Pernicious Anemia.

3. Murphy, W. P., J. A. M. A., 1932, xcviii, 1051. The Parenteral Use of Liver Extract in Pernicious Anemia.

4. Sabin, F. R., Cunningham, R. S., Doan, C. A., and Kindwall, J. A., Bull. Johns Hopkins Hosp., 1925, xxxvii, 14. The Normal Rhythm of the White Blood Cells.

5. Shaw, A. F. B., J. Path. and Bact., 1927, xxx, 1. The Diurnal Tides of the Leukocytes in Man. 